\title{
Molecular clonality and antimicrobial resistance in Salmonella enterica serovars Enteritidis and Infantis from broilers in three Northern regions of Iran
}

\author{
Maral Rahmani ${ }^{1}$, Seyed Mostafa Peighambari ${ }^{1}$, Christina Aaby Svendsen ${ }^{2}$, Lina M Cavaco ${ }^{2}$, Yvonne Agers $\varnothing^{2}$ \\ and Rene S Hendriksen ${ }^{2^{*}}$
}

\begin{abstract}
Background: Multidrug-resistant Salmonella strains are frequently encountered problems worldwide with considerable increased occurrences in recent years. The aim of this study was to investigate the occurrence and frequency of antimicrobial resistance and associated resistance genes in Salmonella isolates from broiler farms in different regions of Iran covering a time period of four years.

Results: From 2007 to 2011, 36 Salmonella strains were isolated from broiler farms located in three northern provinces of Iran. The isolates were serotyped, antimicrobial susceptibility tested, and characterized for antimicrobial resistance genes associated to the phenotype. Pulsed-field gel electrophoresis (PFGE) was applied for comparison of genetic relatedness.

Two serovars were detected among the isolates; Salmonella enterica serovar Infantis (75\%) and S. Enteritidis (25\%). Thirty-four (94\%) of the isolates exhibited resistance to nalidixic acid and ciprofloxacin caused by a single mutation in the quinolone resistance-determining region (QRDR) of gyrA. For all strains this mutation occurred in the codon of Asp ${ }^{87}$ leading to a Asp ${ }^{87}-\mathrm{Tyr}_{\mathrm{r}}$ Asp ${ }^{87-}$ Gly or Asp ${ }^{87}$ Asn substitutions. All $\mathrm{S}$. Infantis $(\mathrm{n}=27)$ were resistant to tetracycline, spectinomycin, streptomycin, and sulfamethoxazole and harbored the associated resistance genes; tetA, dfrA14, aadA1, and sull together with class 1 integrons. The isolates revealed highly similar PFGE patterns indicating clonal relatedness across different geographical locations.
\end{abstract}

Conclusion: The data provided fundamental information applicable when launching future control programs for broilers in Iran with the aim to conserve the effectiveness of important antimicrobials for treatment in humans.

Keywords: Salmonella infantis, Salmonella enteritidis, Antimicrobial resistance, MIC determination, Resistance gene, PFGE, Fluoroquinolone, Poultry, Iran

\section{Background}

Salmonellosis is one of the most important diseases in both humans and animals and has been described as the second most common cause of foodborne bacterial human disease worldwide [1]. It is estimated that 93.8 million cases of gastroenteritis due to Salmonella spp. occur annually worldwide leading to 155,000 deaths each year [2]. A significant increase in the number of

\footnotetext{
* Correspondence: rshe@food.dtu.dk

${ }^{2}$ WHO Collaborating Center for Antimicrobial Resistance in Food borne Pathogens and European Union Reference Laboratory for Antimicrobial Resistance, National Food Institute, Technical University of Denmark, Kgs. Lyngby, Denmark

Full list of author information is available at the end of the article
}

Salmonella infections has been observed in many countries over the past decade [3]. Globally, the most prevalent serovars in humans are Salmonella enterica serovars Typhimurium and Enteritidis [4].

Chicken and related products are recognized as important reservoirs for Salmonella and vehicles for salmonellosis. Some Salmonella serovars such as $S$. Enteritidis, $S$. Infantis, $S$. Kentucky, and $S$. Heidelberg appear to be more prevalent in poultry than in other food animals [5].

Widespread use of antimicrobial agents in food animal production has contributed to the occurrence in animals of resistant zoonotic pathogens such as Salmonella. Whilst antimicrobial resistance is moderate in the predominant 
poultry-related serovar; $S$. Enteritidis [5,6], multidrug resistance (MDR) is frequent in other Salmonella serovars [6]. In poultry production, antimicrobial agents are widely used for growth promotion, prophylaxis or treatment purposes $[7,8]$. As a consequence, chicken and chicken meat can harbor antimicrobial resistant strains and function as a vehicle for dissemination of these to humans. Today, MDR Salmonella strains are frequently encountered in most of the world and the rates of MDR have increased considerably in recent years [9]. As a result, extended-spectrum cephalosporins (ESC) and fluoroquinolones (FQ) are the drugs of choice for treatment of severe Salmonella infections. Accordingly, limiting the occurrence of resistance to ESC and FQ is a public health priority and these drugs have been classified by the World Health Organization as critically important antimicrobials [10]. The resistance mechanisms responsible for the increase in antimicrobial resistance are mainly a result of the horizontal gene transfer via mobile genetics elements such as plasmids and integrons. The strong association between MDR Salmonella and the presence of integrons; especially class 1 , has been well documented [11]. To embark on and enforce prevention and control measurements, it is vital to elucidate and understand the global epidemiology of antimicrobial resistance pheno- and genotypes [12]. In recent years, MDR bacterial pathogens have emerged and disseminated in Iran and have become a challenging problem for the medical and veterinary community. Unfortunately, studies revealing the frequency of antimicrobial resistance and corresponding genes in pathogens of poultry origin are sparse. Poultry is one of the predominant reservoirs for Salmonella in Iran and in the Iranian poultry sector production infrastructure has progressed rapidly in recent years, especially in the northern part of the country.

In this study, the objectives were to investigate the occurrence and frequency of antimicrobial resistance and associated resistance genes in Salmonella isolates from broilers originating from farms located in three northern provinces of Iran in the period from 2007 to 2011 with the application of minimal inhibitory concentration (MIC) determination and polymerase chain reaction (PCR). To elucidate if any genotypes were predominating among the isolates, mechanisms for FQ resistance were investigated by subsequent sequencing of PCR amplicons. Additionally, we utilized pulsed-field gel electrophoresis (PFGE) and MIC patterns to determine the clonal relatedness and to elucidate any epidemiological links between the farms and in a temporal context.

\section{Methods}

Bacterial isolates

A total of 36 broiler-related Salmonella isolates covering a four-year period, from 2007 to 2011, were included in this study. The isolates originated from 14 different broiler farms situated in the northern provinces of Mazandaran, Guilan, and Golestan in Iran. The capacity of the farms ranged from 5,000 to 18,000 broilers per house. Samples taken included fresh randomly collected feces, cloacal feces and a limited number of carcasses at 8 to 49 days old. A randomized convenience sampling was employed and collection, isolation, and identification of Salmonella spp. were conducted according to standard procedures [13].

\section{Serotyping, antimicrobial susceptibility testing, and pulsed-field gel electrophoresis}

The procedures for serotyping and PFGE of the isolates included in this study have been described previously [14]. In brief, all isolates were serotyped using slide agglutination and assigned a serotype according to the Kauffmann-White scheme [15]. In addition, all isolates were genotyped by PFGE using $\mathrm{XbaI}$ according to the CDC PulseNet protocol [16].

MIC determination was performed using a commercially prepared, dehydrated panel, Sensititre, from TREK Diagnostic Systems Ltd. The antimicrobials used and interpretative criteria applied were as follows: ampicillin, AMP ( $>8 \mathrm{mg} / \mathrm{L})$; amoxicillin + clavulanic acid, AUG $(\mathrm{R} \geq 32 \mathrm{mg} / \mathrm{L})$; apramycin, APR $(\mathrm{R}>32 \mathrm{mg} / \mathrm{L})$; cefotaxime, FOT $(\mathrm{R}>0.5 \mathrm{mg} / \mathrm{L})$; ceftiofur, XNL $(\mathrm{R}>2 \mathrm{mg} / \mathrm{L})$; chloramphenicol, CHL (R > $16 \mathrm{mg} / \mathrm{L})$; ciprofloxacin, CIP (R-low level: 0.064 to $1 \mathrm{mg} / \mathrm{L}$, and R-high level > $1 \mathrm{mg} / \mathrm{L}$ ); colistin COL ( $>2 \mathrm{mg} / \mathrm{L})$; florfenicol, FFN ( $>16 \mathrm{mg} / \mathrm{L})$; gentamicin, GEN ( $>2 \mathrm{mg} / \mathrm{L})$; nalidixic acid, NAL ( $\mathrm{R}>16 \mathrm{mg} / \mathrm{L})$; neomycin, NEO ( $\mathrm{R}>4 \mathrm{mg} / \mathrm{L})$; spectinomycin, SPE $(R>64 \mathrm{mg} / \mathrm{L})$; streptomycin, STR $(\mathrm{R}>$ $16 \mathrm{mg} / \mathrm{L})$; sulfamethoxazole, $\mathrm{SMX}(\mathrm{R}>256 \mathrm{mg} / \mathrm{L})$; tetracycline, TET $(\mathrm{R}>8 \mathrm{mg} / \mathrm{L})$; and trimethoprim, TMP ( $\mathrm{R}>2 \mathrm{mg} / \mathrm{L})$. For interpretation of antimicrobial susceptibility test results epidemiological cut-off values according to EUCAST recommendations [17] were applied for all antimicrobials except APR, AUG, SMX, and SPE for which EUCAST values are not available to date. For AUG, SMX and SPE clinical breakpoints according to the Clinical and Laboratory Standards Institute (CLSI) were applied [18-20] and for APR the interpretation was based on research results from DTU Food due to the lack of a CLSI clinical breakpoint. Reference strain E. coli ATCC 25922 was used as a Quality Control according to CLSI standards [18-20].

\section{Screening for resistance genes and integrons}

All isolates exhibiting phenotypic resistance to either of the tested antimicrobials were characterized for the presence of associated antimicrobial resistance genes. Isolates resistant to sulfamethoxazole (all $S$. Infantis) were screened for class 1 and 2 integrons using a PCR 
assay with specific primers. The PCR reactions were performed according to previously described conditions [21]. In brief, three different PCR reactions were performed utilizing the following primers: qacE $\Delta 1-\mathrm{F} /$ qacE $\Delta 1-\mathrm{B}$ targeting qacE $\Delta 1$, SulIB/qacE $\Delta 1-\mathrm{F}$ targeting the sulI and qacE $\Delta 1$ region, and Int $\mathrm{I} 1$ variable- $\mathrm{B}$ targeting the variable region of class 1 integrons. For class 2 integrons, two PCR reactions were used targeting the conserved and the variable region, respectively. Target genes, sequences, annealing temperature, amplicon sizes, and references of all PCR tests are listed in Table 1.

Table 1 Primer sequences used for the amplification of the various resistance genes

\begin{tabular}{|c|c|c|c|c|}
\hline Target genes & Sequence ( $5^{\prime}$ to $\left.3^{\prime}\right)$ & Annealing temp & Size (bp) & Reference \\
\hline \multirow[t]{2}{*}{ tetA } & 5'-GTAATTCTGAGCACTGTCGC-3' & 57 & 950 & [22] \\
\hline & 5'-CTGCCTGGACAACATTGCTT-3' & & & \\
\hline \multirow[t]{2}{*}{ tetB } & 5'-CTCAGTATTCCAAGCCTTTG-3' & 52 & 430 & {$[23]$} \\
\hline & 5'-ACTCCCCTGAGCTTGAGGGG-3' & & & \\
\hline \multirow[t]{2}{*}{ tetC } & 5'-GGTTGAAGGCTCTCAAGGGC-3' & 62 & 505 & [23] \\
\hline & 5'-CCTCTTGCGGGATATCGTCC-3' & & & \\
\hline \multirow[t]{2}{*}{ tetD } & 5'-CATCCATCCGGAAGTGATAGC-3' & 57 & 435 & [24] \\
\hline & 5'-GGATATCTCACCGCATCTGC-3' & & & \\
\hline \multirow[t]{2}{*}{ tetG } & 5'-GCAGCGAAAGCGTATTTGCG-3' & 62 & 680 & [25] \\
\hline & 5'-TCCGAAAGCTGTCCAAGCAT-3' & & & \\
\hline \multirow[t]{2}{*}{ aadA } & 5'-ATTTGCTGGTTACGGTGACC-3' & 56 & 533 & [21] \\
\hline & 5'-CTTCAAGTATGACGGGCTGA-3' & & & \\
\hline \multirow[t]{2}{*}{ strA } & 5'-CCAATCGCAGATAGAAGGC-3' & 55 & 500 & [26] \\
\hline & 5'-CTTGGTGATAACGGCAATTC-3' & & & \\
\hline \multirow[t]{2}{*}{$s t r B$} & 5'-GGATCGTAGAACATATTGGC-3' & 56 & 500 & {$[26]$} \\
\hline & 5'-ATCGTCAAGGGATTGAAACC-3' & & & \\
\hline \multirow[t]{2}{*}{ dfrA14 } & 5'-TGAGAACCTTGAAAGTATCATTG-3' & 55 & 483 & This study \\
\hline & 5'-ACCCTIITCCAAATTTGATAG-3' & & & \\
\hline \multirow[t]{2}{*}{ flork } & 5'-ATGGCAGGCGATATTCATTA-3' & 55 & 320 & This study \\
\hline & 5'-AAACGGGTTGTCACGATCAT-3' & & & \\
\hline \multirow[t]{2}{*}{ sulll } & 5'-GCGCTCAAGGCAGATGGCATT-3' & 70 & 284 & [26] \\
\hline & 5'-GCGTTTGATACCGGCACCCGT-3' & & & \\
\hline \multirow[t]{2}{*}{ sul1/qacE $\Delta 11$} & 5'-ATCGCAATAGTTGGCGAAGT-3' & 58 & 798 & [22] \\
\hline & 5'-GCAAGGCGGAAACCCGCGCC-3 & & & \\
\hline \multirow[t]{2}{*}{ sull } & 5'-TGAGATCAGACGTATTGCGC-3' & 58 & 420 & This study \\
\hline & 5'-TTGAAGGTTCGACAGCACGT-3' & & & \\
\hline \multirow[t]{2}{*}{ qacE $\Delta 1$} & 5'-ATCGCAATAGTTGGCGAAGT-3' & 57 & 226 & [22] \\
\hline & 5'-CAAGCTTTTGCCCATGAAGC-3' & & & \\
\hline \multirow[t]{2}{*}{$\ln t 11$} & 5'-AAGCAGACTTGACCTGAT-3' & 55 & Variable & {$[22]$} \\
\hline & 5'-GGCATCCAAGCAGCAAGC-3' & & & \\
\hline \multirow[t]{2}{*}{$\ln t 12$} & 5'-CACGGATATGCGACAAAAAGGT-3' & 60 & 789 & [27] \\
\hline & 5'-GTAGCAAACGAGTGACGAAATG-3' & & & \\
\hline \multirow[t]{2}{*}{ Int12 variable } & 5'-GACGGCATGCACGATTTGTA-3' & 56 & 2214 & {$[28]$} \\
\hline & 5'-GATGCCATCGCAAGTACGAG-3' & & & \\
\hline \multirow[t]{2}{*}{ gyrA } & 5'-TACCGTCATAGTTATCCACGA-3' & 60 & 312 & [29] \\
\hline & 5'-GTACTITACGCCATGAACGT-3' & & & \\
\hline \multirow[t]{2}{*}{ parC } & 5'-CTATGCGATGTCAGAGCTGG-3' & 59 & 261 & [29] \\
\hline & 5'-TAACAGCAGCTCGGCGTATT-3' & & & \\
\hline
\end{tabular}


All isolates resistant to nalidixic acid and ciprofloxacin (all isolates included in this study with the exception of two $S$. Enteritidis) were tested by PCR amplification to detect possible chromosomal mutations in DNA gyrase $(\operatorname{gyr} \mathrm{A})$ and topoisomerase IV $(\operatorname{parC})$. Amplicons of the genes gyrA and parC were purified using the $\mathrm{GFX}^{\mathrm{m}} \mathrm{PCR}$ DNA kit (Amersham Biosciences, Piscataway, New Jersey, US) and one sample of each serotype per farm was shipped to Macrogen Inc., South Korea for sequencing using the same forward primers as used in the PCR analysis. Vector NTI suite 11 (InforMax, Inc.) software (Bethesda, Maryland, US) was used for sequencing analysis and alignment. The resulting nucleotide sequences were compared to the corresponding sequences obtained from GenBank [30].

\section{Results}

\section{Serotyping}

Twenty-seven (75\%) out of 36 isolates were typed as $S$. Infantis whereas the remaining isolates were $S$. Enteritidis $(25 \%, \mathrm{n}=9)$. The distribution of $S$. Infantis in each province was $90 \%, 82 \%$, and $50 \%$ in Mazandaran, Golestan, and Guilan, respectively, whereas the distribution of S. Enteritidis was 50\%, 18\%, and 10\% in Guilan, Golestan, and Mazandaran, respectively.

\section{Antimicrobial susceptibility testing}

Thirty-four out of 36 isolates tested were resistant to at least two antimicrobial agents out of 17 agents tested (Figures 1 and 2). Two (5.5\%) S. Enteritidis isolates from the same province were susceptible to all tested antimicrobial agents (Figure 2). The highest frequency $(94 \%, \mathrm{n}=34)$ of antimicrobial resistance was observed to nalidixic acid and ciprofloxacin (Figures 1 and 2). Of the serovars identified in this study, $S$. Enteritidis showed less resistance to antimicrobial agents compared to $S$. Infantis. Of $27 S$. Infantis isolates, all were resistant to ciprofloxacin, nalidixic acid, tetracycline, spectinomycin, streptomycin, sulfamethoxazole. In addition, $17(63.0 \%)$ were resistant to trimethoprim, and one (3.7\%) was resistant to florfenicol and chloramphenicol (Figure 1).

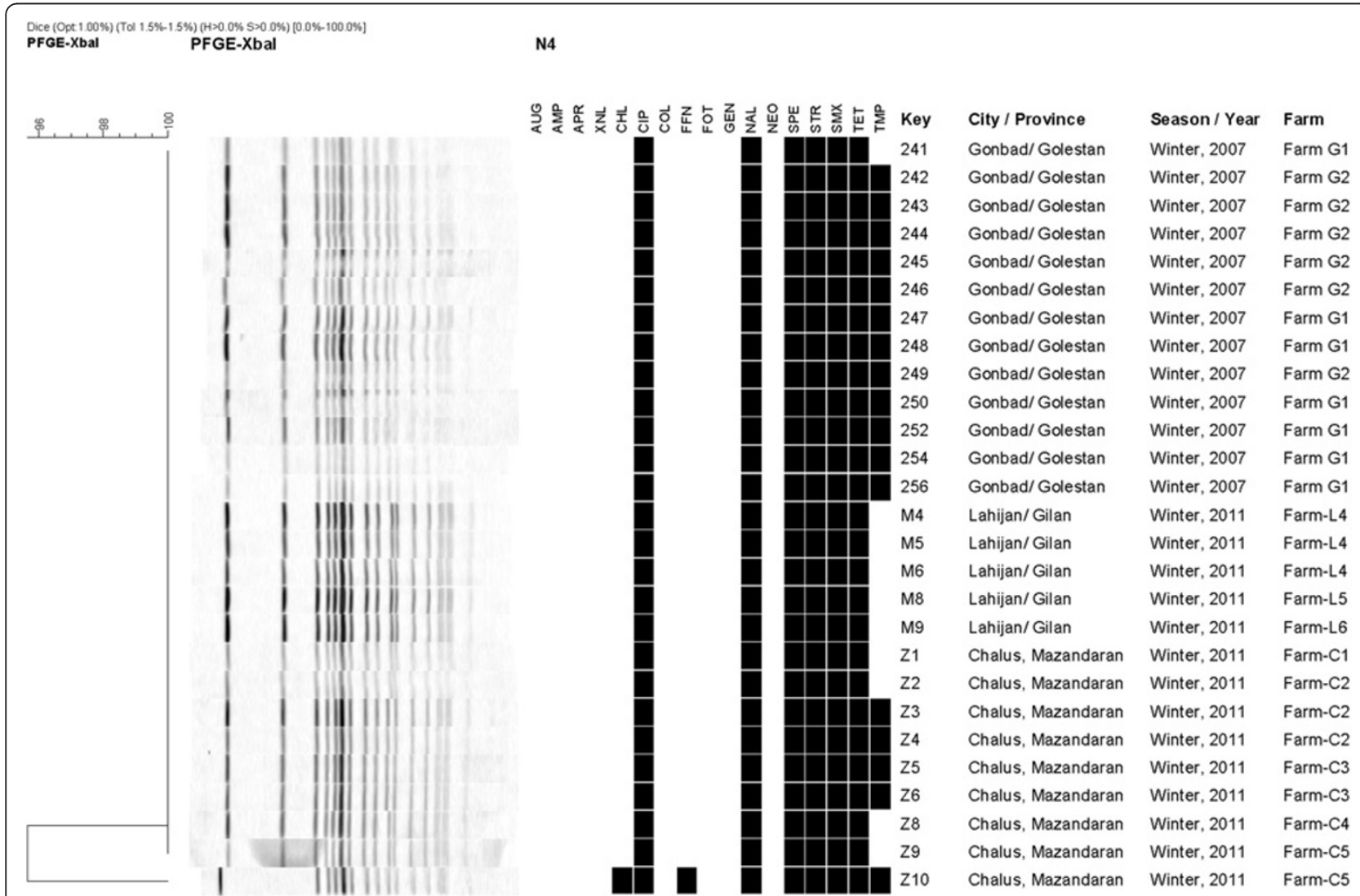

Figure 1 PFGE pattern of Salmonella serovar Infantis isolated from three Northern provinces of Iran. Footnotes: Black squares represent the isolates classified as resistant, Abbreviations: AMP, Ampicillin; AUG, amoxicillin + clavulanic acid; APR, apramycin; FOT, cefotaxime; XNL, ceftiofur; $\mathrm{CHL}$, chloramphenicol; CIP, ciprofloxacin; COL, colistin; FFN, florfenicol; GEN, gentamicin; NAL, nalidixic acid; NEO, neomycin; SPE, spectinomycin; STR, streptomycin; SMX, sulfamethoxazole; TET, tetracycline; TMP, trimethoprim. 


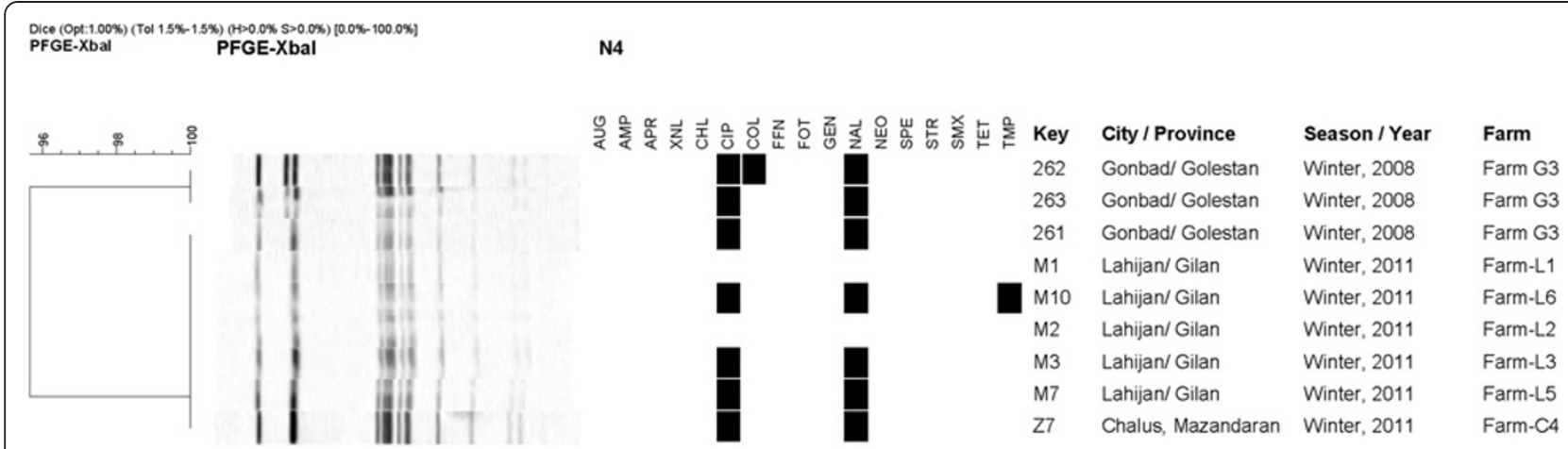

Figure 2 PFGE pattern of Salmonella serovar Enteritidis isolated from three Northern provinces of Iran. Footnotes: Black squares represent the isolates classified as resistant, Abbreviations: AMP, Ampicillin; AUG, amoxicillin + clavulanic acid; APR, apramycin; FOT, cefotaxime; XNL, ceftiofur; CHL, chloramphenicol; CIP, ciprofloxacin; COL, colistin; FFN, florfenicol; GEN, gentamicin; NAL, nalidixic acid; NEO, neomycin; SPE, spectinomycin; STR, streptomycin; SMX, sulfamethoxazole; TET, tetracycline; TMP, trimethoprim.

\section{Screening for resistance genes and integrons}

Out of twenty-seven $S$. Infantis isolates from three provinces, all (100\%) harbored the intl genes confirming the presence of class 1 integrons but none of the $S$. Enteritidis were positive for integron class 1 . The $S$. Infantis isolates were resistant to six or more antimicrobial agents (Figure 1). Seventeen (62.9\%) of the S. Infantis isolates from the provinces of Golestan and Mazandaran carrying the class 1 integron also harbored the class 2 integron, however, the PCR result for the detection of the variable region in integron class 2 was negative. We confirmed the presence of Int $\mathrm{I}$ in two isolates (\#248 and \#Z6) by nucleotide sequence comparison with that of an Escherichia coli strain (GenBank accession no. AU780012) from position 1038 to 1785 and found $100 \%$ similarity. Among the genes conferring resistance to tetracycline, 27 of $S$. Infantis isolates originating from all three provinces carried the tet $\mathrm{A}$ gene. However, none of $S$. Infantis isolates contained tet $\mathrm{B}$, tet $\mathrm{C}$, tet $\mathrm{D}$, and tet $\mathrm{G}$ genes. In addition, no $S$. Infantis isolates were positive for the presence of sulII. S. Infantis isolates that were tested for the presence of strA, strB and aadA1 genes, were positive for aadA1 only. In addition, one $S$. Infantis isolate (2.7\%) from Mazandaran province was found positive for floR. Twenty-two (61.1\%) S. Infantis isolates from the provinces of Golestan and Mazandaran harbored the $d f r A 14$ conferring resistance to trimethoprim whereas only one (2.7\%) S. Enteritidis isolate was positive for $d f r A 14$. The detailed data of all isolates and PCR results for detected genes are shown in Table 2.

Twenty-one (61.7\%) out of 34 isolates that were resistant to nalidixic acid and ciprofloxacin were selected for sequencing and detection of point mutations in the Quinolone Resistance Determining Region (QRDR) of gyrA and parC genes. All of the detected single mutations occurred in codon Asp87 of the gyrA gene. Substitution of Asp-Tyr, Asp-Gly, and Asp-Asn were observed in $13 S$. Infantis (61.9\%), one S. Enteritidis (4.8\%), and seven $S$. Infantis (33.3\%) isolates, respectively. No double mutations were found in the gyrA gene of the tested isolates and no mutations were identified in the parC gene of any of the isolates.

\section{Pulsed-field gel electrophoresis \\ Salmonella Infantis}

Two distinct PFGE patterns were observed among the 27 $S$. Infantis isolates (Figure 1). All but one isolate clustered

Table 2 Distribution of antimicrobial resistance genes of Salmonella isolates originating from poultry in three Northern provinces of Iran

\begin{tabular}{|c|c|c|c|c|c|c|c|c|c|}
\hline \multirow[t]{2}{*}{ Province } & \multirow[t]{2}{*}{ No. of isolates } & \multirow[t]{2}{*}{ Serotype } & \multicolumn{7}{|c|}{ No. and (\%) of isolates with antimicrobial resistance genes } \\
\hline & & & floR & tetA & $\operatorname{aadA1}$ & dfrA14 & sull & $\ln 1$ & $\ln 2$ \\
\hline \multirow[t]{2}{*}{ Golestan } & 13 & S. Infantis & 0 & $13(81)$ & $13(81)$ & $12(75)$ & $13(81)$ & $13(81)$ & $12(75)$ \\
\hline & 3 & S. Enteritidis & 0 & 0 & 0 & 0 & 0 & 0 & 0 \\
\hline \multirow[t]{2}{*}{ Mazandaran } & 9 & S. Infantis & $1(10)$ & $9(90)$ & $9(90)$ & $5(50)$ & $9(90)$ & $9(90)$ & $5(50)$ \\
\hline & 1 & S. Enteritidis & 0 & 0 & 0 & 0 & 0 & 0 & 0 \\
\hline \multirow[t]{2}{*}{ Guilan } & 5 & S. Infantis & 0 & $5(50)$ & $5(50)$ & 0 & $5(50)$ & $5(50)$ & 0 \\
\hline & 5 & S. Enteritidis & 0 & 0 & 0 & $1(10)$ & 0 & 0 & 0 \\
\hline Total & 36 & & 1 (3) & $27(75)$ & $27(75)$ & $18(50)$ & $27(75)$ & $27(75)$ & $17(47)$ \\
\hline
\end{tabular}

All isolates were negative for detection of tetB, tetC, tet $\mathrm{G}, \operatorname{str} \mathrm{A}, \operatorname{str} \mathrm{B}$ and sulll resistance genes. 
within a unique pattern covering all three provinces and the full extent of the tested time period. Sixteen of the indistinguishable isolates within the unique cluster were from the provinces of Golestan and Mazandaran and isolated in 2007 and 2011. Additionally, all of those isolates conferred resistance to the same seven antimicrobials including ciprofloxacin and nalidixic acid (Figure 1). All but one of the remaining ten identical isolates of the unique cluster were isolated in 2011, whereas one isolate was from 2007. The ten isolates were from the province of Golestan $(\mathrm{n}=1)$, Mazandaran $(\mathrm{n}=4)$, and Guilan $(\mathrm{n}=5)$ and all shared the same antimicrobial resistance pattern of six antimicrobials. One isolate; \#Z10 from the province of Mazandaran, demonstrated a unique PFGE and resistance profile compared to the other 26 isolates included this study (Figure 1).

\section{Salmonella enteritidis}

Two clusters were defined for the nine $S$. Enteritidis covering all three provinces and the full extent of the tested time period (Figure 2). One unique pattern comprised seven isolates from all three provinces whereas the second pattern only included two strains from the province of Golestan isolated in 2008 (Figure 2). All nine isolates represented resistance profiles of resistance to ciprofloxacin and nalidixic acid and two isolates additionally conferred resistance to colistin (\#262, Golestan) and trimethoprim (\#M10, Mazandaran) (Figure 2).

\section{Discussion}

Currently, increasing bacterial resistance to antimicrobial agents including quinolones and FQ poses a serious problem throughout the world. To date, several reports from Iran have described the presence of resistance to antimicrobial agents such as FQ and third-generation cephalosporins that are critically important for treatment of infections in humans [31,32].

The widespread overuse and misuse of antimicrobial agents are associated with the development of resistance to these drugs that has emerged as a major problem worldwide. In Iran, patients referred to hospitals with Salmonella infections are usually treated with ciprofloxacin, co-amoxiclav (amoxicillin + clavulanic acid) or cephalosporins [31]. Quinolones and especially FQ are widely used in poultry farms in Iran [33], for example, enrofloxacin, which is chemically closely related to norfloxacin and ciprofloxacin, is one of the antimicrobials most frequently used for the treatment of poultry. Except for a few antimicrobials such as bacitracin and virginamycin, the use of antimicrobials for growth promotion is illegal in Iran, however, one could speculate if growth promotion in some cases might still take place. This might explain the high frequency of resistance to FQ in this study. Unfortunately, there is no surveillance or control system to monitor the prudent use of antimicrobials in the agricultural sector.

A high frequency of resistance to ciprofloxacin and nalidixic acid in $S$. Enteritidis and $S$. Infantis isolated from different regions over time within Iran was found in this study. This correlates with several studies performed in Iran where Morshed and Peighambari [34] observed high levels of resistance to nalidixic acid (24.1\%) and enrofloxacin (6.9\%) among Salmonella isolated from poultry. In comparison, they found lower frequencies of resistance to other antimicrobial drug classes. Rad et al. [33] reported $40 \%$ and $23 \%$ resistance to nalidixic acid and enrofloxacin, respectively, in Salmonella isolates of animal origin. The high resistance rate (94\%) to quinolones observed in this study might indicate common use of enrofloxacin for various reasons during the past decade in veterinary medicine in Iran.

As in the present study, resistance to streptomycin, spectinomycin, tetracycline, trimethoprim, and sulfamethoxazole have also frequently been reported in other studies on poultry products [34-36]. The very low rate of resistance to chloramphenicol among the studied isolates (one $S$. Infantis) may be attributed to its banned use in animal production due to the potential hazards to human consumers' health. In addition, we observed no cephalosporin resistance among the studied isolates which is valuable to the community as cephalosporin resistance is a serious public health concern.

Increased MDR has been reported in Salmonella isolates in many countries including Iran [31,37]. The high level of MDR observed among $S$. Infantis is in agreement with several studies from different countries which have identified healthy poultry as a potential reservoir of $S$. Infantis [35,36,38].

Furthermore, several studies documented the presence of large plasmids in $S$. Infantis carrying antimicrobial resistance (AMR) determinants. Kehrenberg et al. [39] reported transferable plasmid mediated fluoroquinolone resistance (qnrS1) in $S$. Infantis isolates from broilers in Germany. Gal-More et al. and Nogrady et al. [40,41] showed multidrug resistant emerging clones characterized by a large conjugative plasmid harboring the $\operatorname{Tn} 1721$ transposon, including the tet $A$ gene.

In contrast, $S$. Enteritidis was less prone to acquiring resistances than other serotypes as described previously in Spain and other countries [6].

In this study, we describe the presence of class 1 and 2 integrons only attributed to $S$. Infantis. Isolates containing class 1 integrons all conferred resistance to sulfonamides and harbored the sulI resistance gene. Additionally, all antimicrobial resistance phenotypes harbored the following associated resistance genes: streptomycin (aadA1), trimethoprim (dfrA14), florfenicol (floR), and tetracyclines $(\operatorname{tet} \mathrm{A})$. The relationship between the application of 
quinolones and the dissemination of bacterial resistance from animals to humans has been described by several studies $[42,43]$. The results obtained in the current study could be related to those obtained by Tajbakhsh et al. [31] who described Iranian patients infected with $S$. Infantis which were resistant to the same antimicrobial agents and harbored the same resistance determinants as described in this study [31].

Presently, we are in a transition phase where next generation sequencing (NGS) technology is introduced for prediction of resistance phenotypes. Hence, linking resistance genes with resistance phenotypes will be more and more important for the understanding of why some isolates are extremely resistant and why some contain multiple resistance genes encoding resistance to the same antimicrobial classes. Recently, a study described extremely resistant Salmonella Senftenberg isolates harboring an arsenal of resistance genes detected by NGS and the use of a resistance database [44,45]. Many questions are still left unanswered, for example how much each of the genes affects the overall MIC value for the specific antimicrobial class.

Seventeen of the $S$. Infantis isolates also harbored class 2 integrons. For those isolates, the primers utilized for the variable region did not amplify any DNA fragment. This is probably due to not previously observed variations or large insertion in the variable region. Overall, the presence of integrons indicates the important role of those in disseminating resistance determinants in $S$. Infantis. Additionally, this indicates a possible spread of the resistant $S$. Infantis isolates from poultry to humans. Unfortunately, PFGE was not applied in the study of Tajbakhsh et al. why a comparison of PFGE profiles could not be conducted to determine the genetic relatedness.

The serovars of this study predominantly belonged to $S$. Infantis followed by $S$. Enteritidis. EFSA and ECDC [46] have considered $S$. Infantis as the third most common serovar in European patients since 2006 with an increase from $1.0 \%$ to $1.6 \%$ in 2009 in the European Union (EU). Additionally, the same trend has been observed globally, where the overall proportion of $S$. Infantis over the years 2001 to 2007 increased from 1.5\% to 2.2\% [4]. Likewise, chicken meat has been acknowledged as a significant source of $S$. Infantis transmitting the infection to humans [47]. To the best of our knowledge; this is the first study that links $S$. Infantis to poultry in Iran. However, there are several reports in human medicine indicating that $S$. Infantis is an important contributor to human salmonellosis. Hamidian et al. [32] found $20.9 \%$ S. Enteritidis and 5.4\% S. Infantis from 129 Salmonella isolated from humans in Tehran. In addition, the highest level of resistance was observed to nalidixic acid whereas no resistance to ciprofloxacin was observed. The absence of resistance to ciprofloxacin in previous reports most likely reflects the interpretative criteria applied.

All sequenced isolates resistant to nalidixic acid and ciprofloxacin presented a single-mutation in Quinolone Resistance-Determining Regions of gyrA in codon 87. The percentage of strains with mutation in codon 87 is consistent with studies done by San Martin et al. and Liebana et al. [48,49].

No mutation was detected in parC-genes. The high prevalence of $S$. Infantis in this study may suggest an outcome of a clonal expansion and establishment of specific PFGE biotypes of $S$. Infantis. Possibly, $S$. Infantis has developed mechanisms protecting the serovar from major genetic rearrangements or horizontal genetic transfers. Another explanation could be that the serovar has a recent ancestor and therefore broad dissemination has not yet been possible of the limited number of accumulated major evolutionary changes at this point of time [35].

Based on PFGE, indistinguishable patterns were observed among $S$. Enteritidis. The lack of genetic diversity observed for $S$. Enteritidis as determined by PFGE is also shown in previous studies that confirm this serotype as highly clonal [50]. PFGE was of limited value in the epidemiological analysis of these particular isolates, however, this may actually be a reflection of the restricted clonal diversity of pathogenic strains of $S$. Enteritidis [51]. Lack of inclusive studies about serotypes distribution, antimicrobial resistance pattern and molecular investigation of poultry isolates in Iran, make this study unable to obtain confirmatory comparisons of the results. The limitations of this study include a confined geographical coverage and limited number of isolates as well as an overall comparison approach that did not include an indepth molecular characterization of plasmids etc. The combined effect of these is reflected in the research output and it is therefore suggested that future research should take these limitation into consideration when characterizing $S$. Infantis in both humans and poultry.

\section{Conclusion}

The study revealed a high frequency of resistant $S$. Infantis in broilers farmed in three different geographical areas between 2007 and 2011. The isolates especially exhibited resistance to nalidixic acid and ciprofloxacin caused by a point mutation in QRDR of gyrA. Additionally, the isolates harbored class 1 and 2 integrons and contained the $\operatorname{aadA1}, s u l \mathrm{I}$, tetA, and $d f r A 14$ resistance genes. This may suggest widespread misuse or overuse of antimicrobial agents by poultry farmers in Iran. However, the results obtained from serotyping and PFGE patterns are practical for determining the current distribution of MDR serovars of Salmonella and epidemiological state of Salmonella isolates circulating among poultry. This study showed that guidelines are needed in Iran for empiric antibacterial 
therapy based on a local experience of antimicrobial susceptibility testing and restriction of antimicrobial growth promoters and other drugs used without prescription in animals. In addition, the data presented in this study provided valuable fundamental information for future national control programs in Iran to conserve the effectiveness of medically important antimicrobials for treating diseases in humans.

\section{Competing interest}

None of the authors have any conflict of interests.

\section{Authors' contributions}

MR and SMP provided epidemiological data and isolates. SMP, YA and LMC helped to draft the manuscript. MR and CAS carried out the MIC determination, identified antimicrobials resistance genes, and conducted the pulsed field gel electrophoresis. YA and LMC assisted detecting and identifying the integron and fluoroquinolone part of the study. RSH conceived of the study, participated in design, the analysis, and helped to draft the manuscript. MR participated in the design, coordination, conducted the analysis and drafted the manuscript. All authors read and approved the final manuscript.

\section{Acknowledgement}

The authors would like to thank colleagues from the National Food Institute, Technical University of Denmark; Dr. Henrik Hasman for technical advice, Mr. Jacob Dyring Jensen for technical assistance and Ms. Susanne Karlsmose for linguistic assistance. In addition, we would like to thank Iran's Ministry of Science, Research and Technology for financial support provided to Miss Maral Rahmani while staying in Denmark. This work was supported by the World Health Organization Global Foodborne Infections Network (GFN).

\section{Author details}

${ }^{1}$ Department of Clinical Sciences, Faculty of Veterinary Medicine, University of Tehran, Tehran, Iran. ${ }^{2}$ WHO Collaborating Center for Antimicrobial Resistance in Food borne Pathogens and European Union Reference Laboratory for Antimicrobial Resistance, National Food Institute, Technical University of Denmark, Kgs. Lyngby, Denmark.

Received: 10 September 2012 Accepted: 14 March 2013

Published: 5 April 2013

\section{References}

1. Gast RK: Paratyphoid infections. In Diseases of Poultry. 12th edition. Edited by Saif YM, Fadly AM, Glisson JR, McDougald LR, Nolan LK, Swayne DE. Ames: Blackwell Publishing; 2008:636-665.

2. Majowicz SE, Musto J, Scallan E, Angulo FJ, Kirk M, O'Brien SJ, Jones TF, Fazil A, Hoekstra RM: The global burden of nontyphoidal Salmonella gastroenteritis. Clin Infect Dis 2010, 50:882-889.

3. Yang B, Qu D, Zhang X, Shen J, Cui S, Shi Y, Xi M, Sheng M, Zhi S, Meng J: Prevalence and characterization of Salmonella serovars in retail meats of market place in Shaanxi, China. Int J Food Microbiol 2010, 141:63-72.

4. Hendriksen RS, Vieira AR, Karlsmose S, Lo Fo Wong DM, Jensen AB, Wegener HC, Aarestrup FM, et al: Global monitoring of Salmonella serovar distribution World Health Organization Foodborne Infections Network Country Data Bank; results of quality assured laboratories from 2001 to 2007. Foodborne Pathog Dis 2011, 8:887-900.

5. Foley SL, Nayak R, Hanning IB, Johnson TJ, Han J, Ricke SC: Population dynamics of Salmonella enterica serotypes in commercial egg and poultry production. Appl Environ Microbiol 2011, 77:4273-4279. Journal of Antimicrobial Chemotherapyjac.oxfordjournals.org.

6. Alvarez-Fernández E, Alonso-Calleja C, García-Fernández C, Capita R: Prevalence and antimicrobial resistance of Salmonella serotypes isolated from poultry in Spain: Comparison between 1993 and 2006. Int J Food Microbiol 2012, 153:281-287.

7. Gyles CL: Antimicrobial resistance in selected bacteria from poultry. Anim Health Res Rev 2008, 9:149-158.
8. Mathew AG, Cissell R, Liamthong S: Antibiotic resistance in bacteria associated with food animals: A United States perspective of livestock production. Foodborne Pathog Dis 2007, 4:115-133.

9. World Health Organization: Drug-resistant Salmonella. http://www.who.int/ mediacentre/factsheets/fs139/en/.

10. Collignon P, Powers JH, Chiller TM, Aidara-Kane A, Aarestrup FM: World Health Organization ranking of antimicrobials according to their importance in human medicine: a critical step for developing risk management strategies for the use of antimicrobials in food production animals. Clin Infect Dis 2009, 49:132-141.

11. Martinez-Freijo P, Fluit AC, Schmitz FJ, Grek VS, Verhoef J, Jones ME: Class 1integrons in Gram-negative isolates from different European hospitals and association with decreased susceptibility to multiple antibiotic compounds. J Antimicrob Chemother 1998, 42:689-696.

12. Ashtiani MT, Monajemzadeh M, Kashi L: Trends in antimicrobial resistance of fecal Shigella and Salmonella isolates in Tehran, Iran. Indian J Pathol Microbiol 2009, 52:52-55.

13. Waltman WD, Gast RK, Mallinson ET: Salmonellosis. In A Laboratory Manual for the Isolation and Identification of Avian Pathogens. 4th edition. Edited by Swayne DE, Glisson JR, Jackwood MM, Pearson JE, Read WM. Pennsylvania: American Association of Avian Pathologists; 1998:4-13.

14. Hendriksen RS, Le Hello S, Bortolaia V, Pulsrikarn C, Nielsen EM, Pornruangmong S, Chaichana P, Svendsen CA, Weill FX, Aarestrup FM: Characterization of Salmonellaenterica serovar Stanley isolates; a serovar endemic to Asia and associated with travel. J Clin Microbiol 2012, 50:709-720.

15. Grimont PAD, Weill FX: Antigenic formulae of the Salmonella serovars. 9th edition. Paris, France: WHO Collaborating Center for Reference and Research on Salmonella, Institute Pasteur; 2007.

16. Ribot EM, Fair MA, Gautom R, Cameron D, Hunter SB, Swaminathan B, Barrett TJ: Standardization of pulsed-field gel electrophoresis protocols for the subtyping of Escherichia coli 0157:H7, Salmonella, and Shigella for PulseNet. Foodborne Pathog Dis 2006, 3:59-67.

17. The European Committee on Antimicrobial Susceptibility testing -EUCAST. http://www.eucast.org/.

18. Clinical and Laboratory Standards Institute: Performance Standards for Antimicrobial Susceptibility Testing. M100-S16. 18th Informational Supplement. Wayne, PA, USA: CLSI Press; 2008.

19. Clinical and Laboratory Standards Institute: Performance Standards for Antimicrobial Disk and Dilution Susceptibility Tests for bacteria Isolated from Animals. M31-A3. 3rd Edition [Approved Standard]. Wayne, PA, USA; 2008.

20. Clinical and Laboratory Standards Institute: Methods for Dilution Antimicrobial Susceptibility Tests for Bacteria That Grow Aerobically. M07-A7. 7th Edition [Approved Standard]. Wayne, PA, USA; 2006.

21. Hendriksen RS, Bangtrakulnonth A, Pornreongwong S, Pulsrikarn C, Aarestrup FM: Antimicrobial resistance and molecular epidemiology of Salmonella Rissen from animals, food products and patients in Thailand and Denmark. Foodborne Pathog Dis 2008, 5:605-619.

22. Sandvang D, Aarestrup FM, Jensen LB: Characterisation of integrons and antibiotic resistance genes in Danish multiresistant Salmonella enterica Typhimurium DT104. FEMS Microbiol Lett 1997, 157:177-181.

23. Sengelov G, Agerso Y, Halling-Sorensen B, Baloda SB, Andersen JS, Jensen LB: Bacterial antibiotic resistance levels in Danish farmland as a result of treatment with pig manure slurry. Environt Int 2003, 28:587-595.

24. Miranda CD, Kehrenberg C, Ulep C, Schwarz S, Roberts MC: Diversity of tetracycline resistance genes in bacteria from Chilean salmon farms. Antimicrob Agents Chemother 2003, 47:883-888.

25. Agers $\varnothing$ Y, Sandvang D: Class 1 integrons and tetracycline resistance genes in Alcaligenes, Arthrobacterand Pseudomonas spp. Isolated from pigsties and manured soil. Appl Environ Microbiol 2005, 71:7941-7947.

26. Aarestrup FM, Lertworapreecha M, Evans MC, Bangtrakulnonth A, Chalermchaikit T, Hendriksen RS, Wegener HC: Antimicrobial susceptibility and occurrence of resistance genes among Salmonella enterica serovar Weltevreden from different countries. J Antimicrob Chemother 2003, 52:715-718.

27. White PA, Mclver CJ, Rawlinson WD: Integrons and gene cassettes in the Enterobacteriaceae. Antimicrob Agents Chemother 2001, 45:2658-2661.

28. Peirano G, Agersø Y, Aarestrup FM, dos Prazeres Rodrigues D: Occurrence of integrons and resistance genes among sulphonamide-resistant Shigella spp. from Brazil. J Antimicrob Chemother 2005, 55:301-305.

29. Wiuff C, Madsen M, Baggesen DL, Aarestrup FM: Quinolone resistance among Salmonella enterica from cattle, broilers, and swine in Denmark. Microb Drug Resist 2000, 6:11-17. 
30. Lahey Clinic: http://www.lahey.org/studies/.

31. Tajbakhsh M, Hendriksen RS, Nochi Z, Zali MR, Aarestrup FM, Garcia-Migura $L$ : Antimicrobial resistance in Salmonella spp. recoveredfrom patients admitted to six different hospitals in Tehran, Iran from 2007 to 2008. Folia Microbiol 2012, 57:91-97.

32. Hamidian M, Tajbakhsh M, Walther Rasmussen J, Zali MR: Emergence of extended spectrum bata-lactamases in clinical isolates of Salmonellaenterica in Tehran, Iran. Jpn J Infect Dis 2009, 62:368-371.

33. Rad M, Kooshan M, Mesgarani $\mathrm{H}$ : Quinolone resistance among Salmonella enterica and Escherichia coli of animal origin. Comp Clin Pathol 2012, 21:161-165.

34. Morshed R, Peighambari SM: Drug resistance, plasmid profile and random amplified polymorphic DNA analysis of Iranian isolates of Salmonella Enteritidis. New Microbiol 2010, 33:47-56.

35. Hauser E, Tietze E, Helmuth E, Junker E, Prager R, Schroeter A, Rabsch W, Fruth A, Toboldt A, Malorny B: Clonal dissemination of Salmonella enterica serovar Infantis in Germany. Foodborne Pathog Dis 2012, 9:352-360.

36. Nógrády N, Kardos G, Bistyak A, Turcsányi I, Mészáros J, Galántai Z, Juhász A, Samu P, Kaszanyitzky JE, Pászti J, Kiss I: Prevalence and characterization of Salmonella Infantis isolates originating from different points of the broiler chicken-human food chain in Hungary. Int J Food Microbiol 2008, 127:162-167.

37. Madadgar O, Tadjbakhsh H, Salehi TZ, Mahzounieh M, Feizabadi MM: Evaluation of random amplified polymorphic DNA analysis and antibiotic susceptibility applicationin discrimination of Salmonella Typhimurium isolates in Iran. New Microbiologica 2008, 31:211-216.

38. Shahada F, Chuma T, Tobata T, Okamoto K, Sueyoshi M, Takase K: Molecular epidemiology of antimicrobial resistance among Salmonella enterica serovar Infantis from poultry in Kagoshima, Japan. Int J Antimicrob Agents 2006, 28:302-307.

39. Kehrenberg C, Friederichs S, de Jong A, Michael GB, Schwarz S: Identification of the plasmid-borne quinolone resistance gene qnrS in Salmonella enterica serovar Infantis. J Antimicrob Chemother 2006, 58:18-22.

40. Gal-Mor O, Valinsky L, Weinberger M, Guy S, Jaffe J, Schorr YI, Raisfeld A, Agmon V, Nissaan I: Multidrug-resistant Salmonella enterica serovar Infantis in Israel. Emerging Infect Dis 2010, 16:1754-1757.

41. Nógrády N, Tóth A, Kostyák A, Pászti J, Nagy B: Emergence of multidrug resistant clones of Salmonella Infantis in broiler chickens and humans in Hungary. J Antimicrob Chemother 2007, 60:645-648.

42. Hummel R, Tschape H, Witte W: Spread of plasmid-mediated nourseothricin resistance due to antibiotic use in animal husbandry. J Basic Microbiol 1996, 26:461-466.

43. Fábrega A, Sánche-Céspedes J, Soto S, Vila J: Quinolone resistance in the food chain. Int J Antimicrob Agents 2008, 31:307-315.

44. Hendriksen RS, Joensen KG, Lukwesa-Musyani C, Kalondaa A, Leekitcharoenphon P, Nakazwe R, Aarestrup FM, Hasman H, Mwansa JC: Extremely Drug-Resistant Salmonella enterica Serovar Senftenberg Infections in Patients in Zambia. J Clin Microbiol 2013, 51:284-286.

45. Zankari E, Hasman H, Cosentino S, Vestergaard M, Rasmussen S, Lund O, Aarestrup FM, Larsen MV: Identification of acquired antimicrobial resistance genes. J Antimicrob Chemother 2012, 67:2640-2644.

46. European Food Safety Authority, European Centre for Disease Prevention and Control: The European Union Summary Report on Trends and Sources of Zoonoses, Zoonotic Agents and Food-borne Outbreaks in 2009. EFSA Journal 2011, 9:1-338.

47. Noda T, Murakami K, Ishiguro Y, Asai T: Chicken meat is an infection source of Salmonella serovar Infantis for humans in Japan. Foodborne Pathog Dis 2010, 7:727-735.

48. San Martın B, San Martın B, Lapierre L, Toro C, Bravo V, Cornejo J, Hormazabal JC, Borie C: Isolation and molecular characterization of quinolone resistant Salmonella spp. from poultry farms. Vet Microbiol 2005, 110:239-244

49. Liebana E, Clouting C, Cassar C, Randall L, Walker R, Threlfall E, CliftonHadley F, Ridley A, Davies R: Comparison of gyrA mutations, cyclohexane resistance, and the presence of class I integrons in Salmonella enterica from farm animals in England and Wales. J Med Microbiol 2002, 40:1481-1486
50. Hendriksen RS, Hyytia-Trees E, Pulsrikarn C, Pornruangmong S, Chaichana P, Svendsen CA, Ahmed R, Mikoleit M: Characterization of Salmonella enterica serovar Enteritidis isolates recovered from blood and stool specimens in Thailand. BMC Microbio/ 2012, 12:92

51. Thong KL, Ngeow YF, Altwegg M, Navaratnam P, Pang T: Molecular analysis of Salmonella Enteritidis by pulsed-field gel electrophoresis and ribotyping. J Clin Microbiol 1995, 33:1070-1074.

doi:10.1186/1746-6148-9-66

Cite this article as: Rahmani et al:: Molecular clonality and antimicrobial resistance in Salmonella enterica serovars Enteritidis and Infantis from broilers in three Northern regions of Iran. BMC Veterinary Research 2013 9:66.

\section{Submit your next manuscript to BioMed Central and take full advantage of:}

- Convenient online submission

- Thorough peer review

- No space constraints or color figure charges

- Immediate publication on acceptance

- Inclusion in PubMed, CAS, Scopus and Google Scholar

- Research which is freely available for redistribution

Submit your manuscript at www.biomedcentral.com/submit
C) BioMed Central 